\title{
Microstructures and Properties of High-strength Alloys Severely Deformed by Machining
}

\author{
Manabu TANAKA, ${ }^{1)}$ Ryuichi KATO, ${ }^{21}$ Jun-ya KOBAYASHII ${ }^{1)}$ and Rika YODA ${ }^{21}$ \\ 1) Department of Mechanical Engineering, Faculty of Engineering and Resource Science, Akita University, 1-1 Tegatagakuen- \\ cho, Akita 010-8502 Japan. $\quad$ 2) Technology Department, Electronics Division, Kobelco Research Institute Inc., 1-5-5 \\ Takatsuka-dai, Nishi-ku, Kobe 651-2271 Japan.
}

(Received on December 25, 2007; accepted on March 14, 2008)

\begin{abstract}
Microstructures and properties were examined on the chip specimens severely deformed to a given shear strain $(\gamma)$ by machining in the high-strength Ni-base Inconel X-750 alloy and 6061-T6 aluminum alloy. Chip specimens has a deformation structure principally composed of elongated grains (for example, a few $\mu \mathrm{m}$ to about $100 \mu \mathrm{m}$ in length and about $1 \mu \mathrm{m}$ to a few tens $\mu \mathrm{m}$ in width for the chip specimen of $\gamma \approx 3$ of the Inconel X-750 alloy). Many subgrains separated by small or medium angle grain boundaries (misorientation, $\theta<15^{\circ}$ ) were also observed within relatively large grains. In the case where detailed analysis by FESEM/EBSP was possible, the fraction of large angle grain boundaries $\left(\theta \geq 15^{\circ}\right)$ and that of medium angle grain boundaries $\left(5^{\circ} \leq \theta<15^{\circ}\right)$ were relatively small, and most of grain boundaries were small grain boundaries $\left(1^{\circ} \leq \theta<5^{\circ}\right)$ in the chip specimens of both alloys. Internal friction of the chip specimen was much larger than that of the original material. Large internal friction of the chip specimens was principally attributed to many cracks formed during machining. The hardness of chip specimens largely increased with increasing shear strain imposed by machining in the Inconel X-750 alloy, although the increase of hardness with shear strain was not so large in the 6061-T6 alloy.
\end{abstract}

KEY WORDS: microstructure; FESEM/EBSP method; machining; internal friction.

\section{Introduction}

Severe plastic deformation techniques such as equal channel angular pressing (ECAP), ${ }^{1-3)}$ high pressure torsion $(\mathrm{HPT})^{4)}$ and accumulative roll-bonding $(\mathrm{ARB})^{5)}$ have recently drawn considerable attention to the production of nano-structured materials, since nano-structured materials sometimes show very high strength without serious loss of ductility. ${ }^{6,7)}$ R. R. Mulyukov et al. ${ }^{8)}$ and Y. Koizumi et $a l{ }^{9,10)}$ reported that ultra-fine grained metals have large internal friction together with high strength, although highstrength materials generally have low damping capacity (internal friction). ${ }^{11,12)}$ Interesting properties of nano-structured materials are related to the largely increased density of lattice defects such as large angle grain boundaries and dislocations. ${ }^{6,8-10,13-15)}$ However, application of these techniques is limited to relatively low-strength materials. T. L. Brown et al. ${ }^{16)}$ and S. Swaminathan et al. ${ }^{17)}$ proposed a lowcost cutting process for production of nano-structured metals and alloys with very high hardness. A large shear strain $(\gamma)$ up to about 20 can be imposed on chips by a single cutting process. This process is also applicable to the production of nano-crystals in high-strength materials. According to the selected area electron diffraction patterns of thin films of the 6061-T6 alloy using a transmission electron microscope, M. Ravi Shankar et al. ${ }^{18)}$ concluded qualitatively that there exists a small region containing high angle boundaries in the chip specimens deformed to a shear strain up to 3.2 or 5.2. However, it is still unclear whether grains in chip specimens are principally surrounded by large angle grain boundaries as in nano-materials produced by other processes, since detailed quantitative analysis of microstructures were not carried out on chip specimens produced by machining. Further, there seems to be no reports on the internal friction of chip specimens.

In this study, microstructures were examined on the chip specimens produced by machining of Inconel X-750 alloy and 6061-T6 aluminum alloy. These alloys were chosen by the following reasons. Inconel X-750 alloy is a representative high-strength nickel-base heat-resistant alloy to which a large plastic strain is difficult to be imposed by other methods such as ECAP, HPT and ARB. Further, it seems to be necessary to carry out quantitative analysis of grain boundary misorientations between grains to make clear the grain structures of chip specimens in 6061-T6 alloy. Misorientations between grains in chip specimens were examined by orientation imaging microscopy (OIM), ${ }^{19,20)}$ namely, by the electron backscatter diffraction pattern (EBSP) method using a field emission scanning electron microscope (FESEM). Internal friction and hardness of chip specimens were measured on the chip specimens deformed to certain shear strains by machining. The mechanism of the internal friction in the chip specimens was also discussed on the basis of the microstructural observation. 
Table 1. Chemical composition of the alloys used in this study (wt \%).

\begin{tabular}{|c|c|c|c|c|c|c|c|c|c|c|c|c|c|c|}
\hline Alloys & $\mathrm{C}$ & $\mathrm{Cr}$ & $\mathrm{Ni}(+\mathrm{Co})$ & $\mathrm{Nb}+\mathrm{Ta}$ & $\mathrm{Ti}$ & $\mathrm{Al}$ & $\mathrm{Fe}$ & $\mathrm{Cu}$ & $\mathrm{Mn}$ & $\mathrm{Si}$ & $\mathrm{Mg}$ & $\mathrm{Zn}$ & $\mathrm{S}$ & $\mathrm{P}$ \\
\hline Inconel X-750 & 0.06 & 15.03 & 71.80 & 1.01 & 2.43 & 1.26 & 7.48 & 0.13 & 0.31 & 0.26 & - & - & 0.001 & 0.007 \\
\hline $6061-\mathrm{T} 6$ & - & 0.08 & - & - & 0.04 & Bal. & 0.36 & 0.22 & 0.03 & 0.64 & 0.86 & 0.07 & - & - \\
\hline
\end{tabular}

Table 2. Cutting conditions and geometry of resulting chip specimens of the alloys in machining.

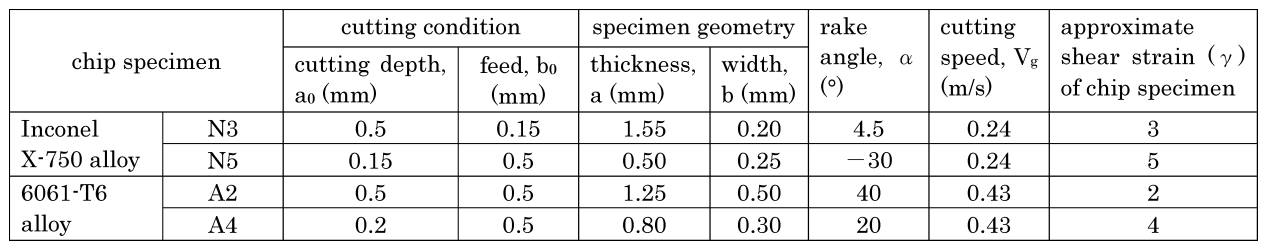

\section{Experimental Procedure}

\subsection{Production of Chip Specimens}

Table 1 lists the chemical composition of non-ferromagnetic Inconel X-750 nickel-base alloy and 6061-T6 aluminum alloy used in this study. Inconel X-750 alloy bars of $20 \mathrm{~mm}$ diameter were solution-heated for $7.2 \mathrm{ks}$ at $1423 \mathrm{~K}$ to give an average grain diameter of $108 \mu \mathrm{m}$. The as received 6061-T6 alloy bars of $30 \mathrm{~mm}$ diameter have an average grain diameter of $80 \mu \mathrm{m}$. The alloy bars were then machined to produce chip specimens deformed to a certain shear strain by a lathe using cutting tools of different rake angles $\left(\alpha=40^{\circ}\right.$ to $\left.-30^{\circ}\right)$ with cutting fluid. Table 2 lists the cutting conditions and geometry of resulting chip specimens in machining. Figure 1 shows the schematic illustration of cutting process and the geometry of chip specimens. Relatively low cutting speed $\left(V_{\mathrm{g}}=0.24 \mathrm{~m} / \mathrm{s}\right.$ or $\left.0.43 \mathrm{~m} / \mathrm{s}\right)$ was chosen to avoid excessive heating of chip specimens during machining. The table also shows the cutting depth $\left(a_{0}\right)$ and feed $\left(b_{0}\right)$ in machining, and the thickness $(a)$ and width $(b)$ of the resulting chip specimens. Chip specimens are designated by N3 or N5 specimen for Inconel X-750 alloy and by $\mathrm{A} 2$ or A4 according to shear strain imposed by machining in this study. The approximate shear strain $(\gamma)$ of the chip specimens was calculated by the following theoretical equation for two-dimensional cutting, ${ }^{21,22)}$ although the cutting condition was not two-dimensional for producing long $\mathrm{N} 3$ and A2 specimens (more than about $20 \mathrm{~mm}$ in length).

$$
\gamma=\cot \phi+\tan (\phi-\alpha)
$$

where $\phi$ is the shear plane angle and

$$
\left.\tan \phi=\left(a_{0} / a\right) \cos \alpha /\left[1-\left(a_{0} / a\right) \sin \alpha\right]\right)
$$

Figure 2 shows the scanning electron micrographs of N3 chip specimen (shear strain, $\gamma \approx 3$ ) of Inconel X-750 alloy. One side of the chip specimen has smooth surface (Fig. 2(a)) and the other side has rough surface with the maximum height difference of about $50 \mu \mathrm{m}$ (Fig. 2(b)). Chip specimens have many cracks caused by shear deformation during machining (indicated by arrows in Fig. 2). Similar cracks were also observed in the chip specimens of the 6061-T6 alloy. The solution-treated (Inconel X-750 alloy) and the as received (6061-T6 alloy) specimens ( $1 \mathrm{~mm} \times$ $5 \mathrm{~mm} \times 20 \mathrm{~mm}$ ) was also used for comparison in the internal friction measurements. Further, the effect of annealing for $3.6 \mathrm{ks}$ at $973 \mathrm{~K}$ on the microstructure, internal friction and hardness was examined on the chip specimens of the Inconel X-750 alloy deformed to a shear strain of about 3 . (a)

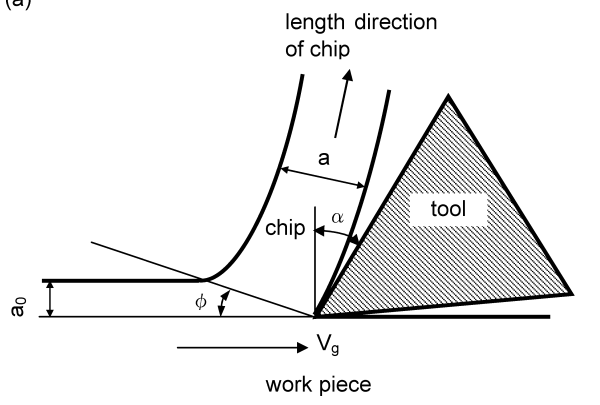

(b)

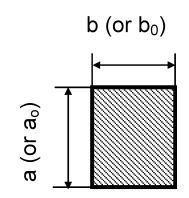

Fig. 1. Schematic illustration of cutting process and geometry of chip specimens. (a) Cutting process, (b) cross section of chip specimens. $a_{0}$ is cutting depth and $b_{0}$ is feed in machining, $a$ is thickness and $b$ is width of chip specimens, $V_{\mathrm{g}}$ is cutting speed, $\phi$ is shear angle, and $\alpha$ is rake angle.

\subsection{Analysis of Microstructures}

Misorientation (rotation angle, $\theta^{\circ}$ ) between neighbouring grains in the chip specimens was examined by the electron backscatter diffraction pattern (EBSP) method $^{19,20)}$ using a field emission scanning electron microscope (FESEM) (or simply, FESEM/EBSP method). The analyzed area was typically $100 \mu \mathrm{m} \times 100 \mu \mathrm{m}$ in the central part of the chip specimen surface (the surface parallel to the thickness-length plane of the chip specimen, as shown in Fig. 1), while much smaller area $(10 \mu \mathrm{m} \times 10 \mu \mathrm{m})$ was also analyzed in some chip specimens. This is orientation imaging microscopy (OIM). Boundaries with the misorientation $\left(\theta^{\circ}\right)$ equal to or larger than $1^{\circ}$ are defined as "grain boundaries" in this study. It is conventionally defined that grain boundaries with the misorientation $\theta \geq 15^{\circ}$ are large angle grain boundaries and those with the misorientation $1^{\circ} \leq \theta<5^{\circ}$ are small angle grain boundaries, although the boundaries with the misorientation $5^{\circ} \leq \theta<15^{\circ}$ are classified into medium angle grain boundaries. Further, the regions surrounded by large angle grain boundaries $\left(\theta \geq 15^{\circ}\right)$ are defined as "grains" and those separated by small or medium grain boundaries $\left(\theta<15^{\circ}\right)$ are defined as "subgrains" in this study. The fractions of large, medium and small angle grain boundaries are also examined in some chip specimens. Microstructures of the chip specimens (severely deformed specimens) were examined using both optical microscope and scanning electron microscope. The specimens for metallographic observation were mechanically polished using diamond paste (the grain size is 3 to $0.25 \mu \mathrm{m}$ ) and were then electrolytically etched using $10 \%$ chromium acid-water solution. The hardness of the chip specimens and the as solution-treated 

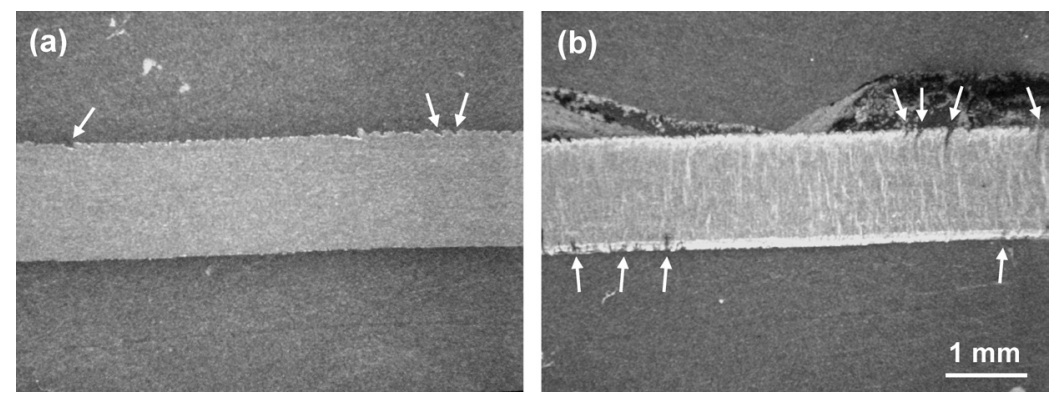

Fig. 2. Scanning electron micrographs of the N3 chip specimen (shear strain, $\gamma \approx 3$ ) of the Inconel X-750 alloy. (a) Smooth surface, (b) rough surface (reverse side). Arrows indicate cracks.
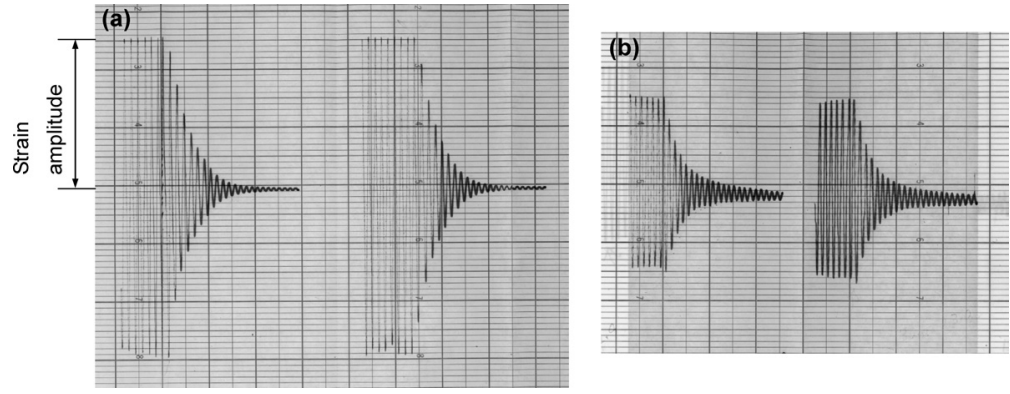

Fig. 3. Examples of the change in the strain amplitude during measurements of internal friction in the $\mathrm{N} 3$ chip specimen $(\gamma \approx 3)$ of the Inconel X-750 alloy. (a) Strain amplitude is $8.60 \times 10^{-4}$, (b) strain amplitude is $7.33 \times 10^{-5}$.

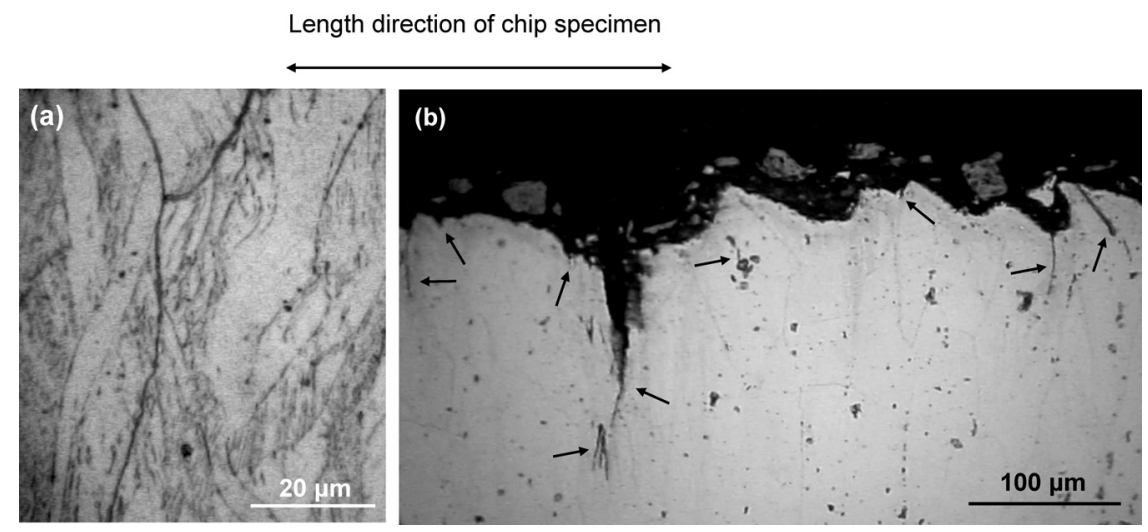

Fig. 4. Microstructures of the N3 chip specimen $(\gamma \approx 3)$ of the Inconel $X-750$ alloy. (a) Interior of the specimen, (b) near the specimen surface. Microcracks are indicated by arrows.

specimens was measured using a Micro Vickers Hardness Tester at the load of $0.98 \mathrm{~N}$. The hardness is an averaged value over ten indentations in the same specimen. The solution-treated specimens for hardness tests were electrolytically polished using $20 \%$ perchloric acid- $10 \%$ glycerin-70\% ethyl alcohol solution.

\subsection{Internal Friction Measurements}

The internal friction of chip specimens was measured by the free decay method using the torsional pendulum-type equipment at room temperature. The (torsional) strain amplitude was in the range from about $2 \times 10^{-6}$ to $1.2 \times 10^{-3}$ and the frequency in the measurements was between 0.19 to $0.50 \mathrm{~Hz}$ for the chip specimens. Figure 3 shows examples of the change in the strain amplitude during the measurements of internal friction in the N3 chip specimens $(\gamma \approx 3)$ of the Inconel X-750 alloy. The strain amplitude $(\varepsilon)$ in the measurement was calculated from the reading on the chart of a recorder. Decay of free oscillation depends on the strain amplitude of the measurement (the strain amplitude is shown in different scales in Figs. 3(a) and 3(b)). The value of internal friction, $Q^{-1}$, was calculated using the following equation ${ }^{23,24)}$ :

$$
Q^{-1}=\delta / \pi=(1 / m \pi) \ln \left(A_{1} / A_{m+1}\right) .
$$

where $A_{1}$ is the strain amplitude of the first free oscillation, $A_{m+1}$ is that of the $(m+1)$ th free oscillation and $\delta$ is the logarithmic decrement. The value of $m$ is chosen to be 10 or 20 in this study. The internal friction in the as solution-treated or as received specimens was also measured in the strain amplitude range from $1 \times 10^{-5}$ to $6 \times 10^{-3}$ at the frequency of 4.2 to $4.4 \mathrm{~Hz}$.

\section{Results and Discussion}

\subsection{Microstructures of Chip Specimens}

3.1.1. Inconel X-750 Alloy

Figure 4 shows the microstructure of the N3 chip speci- 
men $(\gamma \approx 3)$ of the Inconel X-750 alloy. The length direction of the specimen is horizontal. Figure 4(a) shows elongated grains lying transversely to the length direction. Deeply etched grain boundaries seem to be large angle grain boundaries. There are many microcracks near the surface of the specimen (indicated by arrows in Fig. 4(b)). These microcracks are identical to those shown in Fig. 2, which were formed by shear deformation during machining. Orientation difference between grains in the chip specimens was examined by the FESEM/EBSP method. Figure 5 shows the image quality map and inverse pole figure map showing the crystal orientations parallel to ND (normal direction to specimen surface) of the N3 chip specimen $(\gamma \approx 3)$ of the Inconel X-750 alloy. Grain boundaries exhibit dark contrast in the image quality map (Fig. 5(a)). The length direction of the chip specimen (the direction of chip formation) is vertical. The analyzed area is $100 \mu \mathrm{m} \times 100 \mu \mathrm{m}$ (Fig. 5(a)). As known from the inverse pole figure (Fig. 5(b)), the N3 chip specimen has a deformation structure principally composed of elongated grains of a few $\mu \mathrm{m}$ to about $100 \mu \mathrm{m}$ in length (about $1 \mu \mathrm{m}$ to a few tens $\mu \mathrm{m}$ in width), which were produced by shear deformation as in the case of microcracks (Fig. 4). These elongated grains lie approximately normal to the length direction of the chip specimen. Figure 6 shows the boundary misorientation map and the fraction of grain boundaries with different misorientations $(\theta)$ in the N3 chip specimen $(\gamma \approx 3)$ of the Inconel X-750 alloy. Grain boundaries with different misorientations $(\theta)$ are shown in different colors in the figure. Many subgrains separated by small or medium angle grain boundaries $\left(\theta<15^{\circ}\right)$ are also observed within relatively large grains. The fraction of large angle grain boundaries $\left(\theta \geq 15^{\circ}\right)$ is about $9 \%$, that of medium angle grain boundaries $\left(5^{\circ} \leq \theta<15^{\circ}\right)$ is about $16 \%$ and most of grain boundaries are small angle grain boundaries $\left(1^{\circ} \leq \theta<5^{\circ}\right)$ (Fig. 6(b)). Some of fine grains surrounded by large angle grain boundaries may be residual precipitates.

Figure 7 shows the image quality map and inverse pole figure map showing the crystal orientations parallel to ND of the N5 chip specimen $(\gamma \approx 5)$ of the Inconel X-750 alloy. The length direction of the chip is vertical. The analyzed area is $10 \mu \mathrm{m} \times 10 \mu \mathrm{m}$ (Fig. 7(a)). The specimen principally consists of elongated grains of submicron to a few $\mu \mathrm{m}$ in length (Fig. 7(b)). The N5 chip specimen seems to involve more large angle grain boundaries compared with the N3 chip specimen $(\gamma \approx 3)$. Swaminathan et al. ${ }^{17)}$ found by transmission electron microscopy of thin films that the average misorientation between grains increased with increasing shear strain of chip specimens in OFHC $\mathrm{Cu}$. As shown in Fig. 8, many subgrains separated by small or medium angle grain boundaries $\left(\theta<15^{\circ}\right)$ exist within relatively large grains surrounded by large angle grain boundaries $\left(\theta \geq 15^{\circ}\right)$. Nanoscale equiaxed grains which were considered as a result of dynamic recrystallization, were observed in the chips of OFHC $\mathrm{Cu}^{17)}$ and in those of 6061-T6 alloy ${ }^{18)}$ by transmission electron microscopy (TEM) of thin films. Unfortunately, the resolution limit of FESEM/EBSP method is considered to be on the order of $0.1 \mu \mathrm{m}$ due to high dislocation density (large strain) in chip specimens, whereas the resolution limit is usually around $0.02 \mu \mathrm{m}$. Therefore, detailed quantitative data could not be obtained in the N5 specimen, because the smallest grain size was close to the resolution limit of FESEM/EBSP method in this study.

Figure 9 shows the image quality map and inverse pole figure map showing the crystal orientations parallel to ND of the chip specimen $(\gamma \approx 3)$ of the Inconel X-750 alloy annealed for $3.6 \mathrm{ks}$ at $973 \mathrm{~K}$. The length direction of the chip specimen is vertical. The analyzed area is $100 \mu \mathrm{m} \times 100 \mu \mathrm{m}$ (Fig. 9(a)). Many elongated grains close to [001] or [111] orientation lie approximately normal to the length direction of the chip specimen (Fig. 9(b)). Microstructures in the annealed chip specimen $(\gamma \approx 3)$ are essentially the same as those in the N3 chip specimen. Ni and Alpas ${ }^{25)}$ observed the recrystallization in the annealing of the chip specimens produced by dry machining of copper at relatively low temperature range (434 to $474 \mathrm{~K}$ ). The annealing temperature $(973 \mathrm{~K})$ is considered high enough to cause recrystallization in the chip specimens of the Inconel X-750 alloy. Microscopic observation using a scanning electron microscope revealed that fine precipitates of $\gamma^{\prime}$ phase or $\mathrm{M}_{23} \mathrm{C}_{6}$ carbide $^{26)}$ whose size was smaller than about $0.2 \mu \mathrm{m}$ were formed on grain boundaries and in the grains of the annealed chip specimen. Excessive vacancies as well as many dislocations introduced by machining may promote the formation of these precipitates during annealing. These fine precipitates may retard the recrystallization in the chip specimen, although dislocation density may decrease by annihilation of dislocations during annealing. Figure 10 shows the fraction of grain boundaries with different misorientations $(\theta)$ in the chip specimen $(\gamma \approx 3)$ of the Inconel $\mathrm{X}-750$ alloy annealed for $3.6 \mathrm{ks}$ at $973 \mathrm{~K}$. As in the N3 chip specimen without annealing, many subgrains were observed within relatively large grains. The fraction of large angle grain boundaries $\left(\theta \geq 15^{\circ}\right)$ is about $14 \%$ and that of medium angle grain boundaries $\left(5^{\circ} \leq \theta<15^{\circ}\right)$ is about $20 \%$. Both values are larger than those of the chip specimen without annealing (about 9\%) (Fig. 6(b)), although most of grain boundaries are small angle grain boundaries $\left(1^{\circ} \leq\right.$ $\left.\theta<5^{\circ}\right)$.

\subsubsection{1-T6 Alloy}

Elongated grains as well as many microcracks formed during machining were also observed in the chip specimens of the 6061-T6 aluminum alloy. Figure 11 shows the image quality map and inverse pole figure map showing the crystal orientations parallel to ND of the A4 chip specimen $(\gamma \approx 4)$ of the 6061-T6 alloy. Grain boundaries exhibit dark contrast in the image quality map (Fig. 11(a)). The length direction of the chip specimen (the direction of chip formation) is vertical. The analyzed area is $100 \mu \mathrm{m} \times 100 \mu \mathrm{m}$ also in this case (Fig. 11(a)). The chip specimen of the 6061-T6 alloy has a deformation structure similar to that of the Inconel X-750 alloy (Fig. 6). The specimen shows a mixed grain structure composed of elongated grains of a few $\mu \mathrm{m}$ to a few tens $\mu \mathrm{m}$ in length (about $1 \mu \mathrm{m}$ to $10 \mu \mathrm{m}$ in width) lying transversely to the length direction of the chip specimen (Fig. 11(b)). Figure 12 shows the boundary misorientation map and the fraction of grain boundaries with different misorientations $(\theta)$ in the A4 chip specimen $(\gamma \approx 4)$ of the 6061-T6 alloy. Grain boundaries with different misorientations $(\theta)$ are shown in different colors (Fig. 12(a)). As 


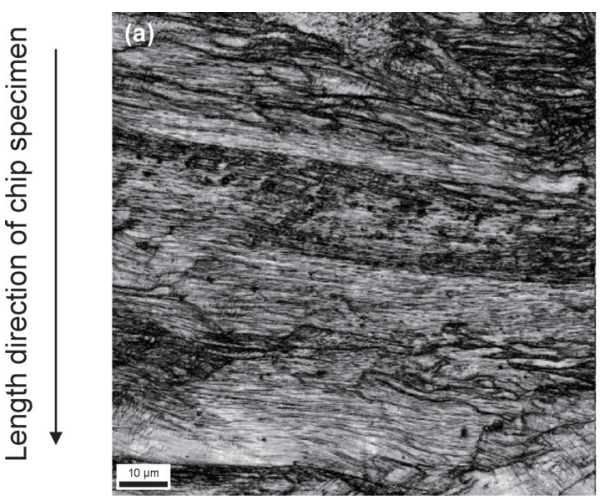

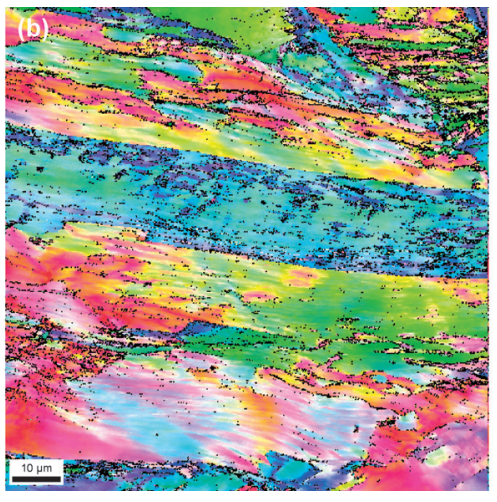

Fig. 5.

Image quality map and inverse pole figure map showing the crystal orientations parallel to ND (normal direction to specimen surface) of the N3 chip specimen $(\gamma \approx 3)$ of the Inconel $\mathrm{X}-750$ alloy.

(a) Image quality map, (b) inverse pole figure map (color coded map).

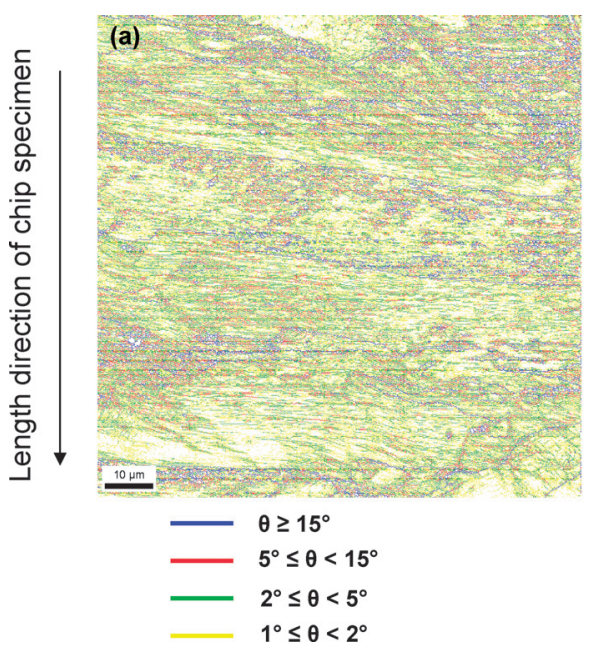

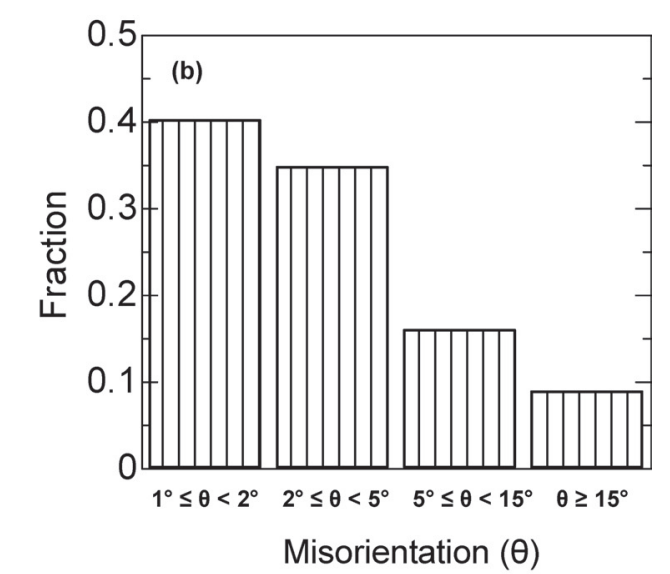

Misorientation $(\theta)$

Fig. 6.

Boundary misorientation map and fraction of grain boundaries with different misorientations $(\theta)$ in the $\mathrm{N} 3$ chip specimen $(\gamma \approx 3)$ of the Inconel X-750 alloy. (a) Boundary misorientation map, (b) fraction of grain boundaries with different misorientations $(\theta)$
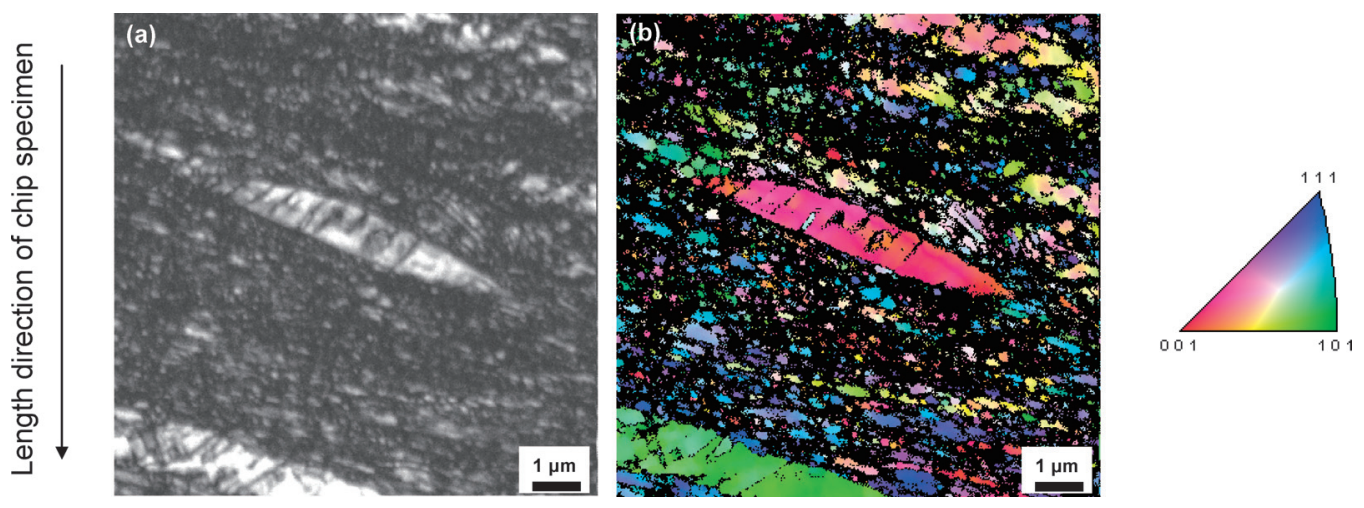

Fig. 7.

Image quality map and inverse pole figure map showing the crystal orientations parallel to ND of the N5 chip specimen $(\gamma \approx 5)$ of the Inconel X-750 alloy.

(a) Image quality map, (b) inverse pole figure map (color coded map).

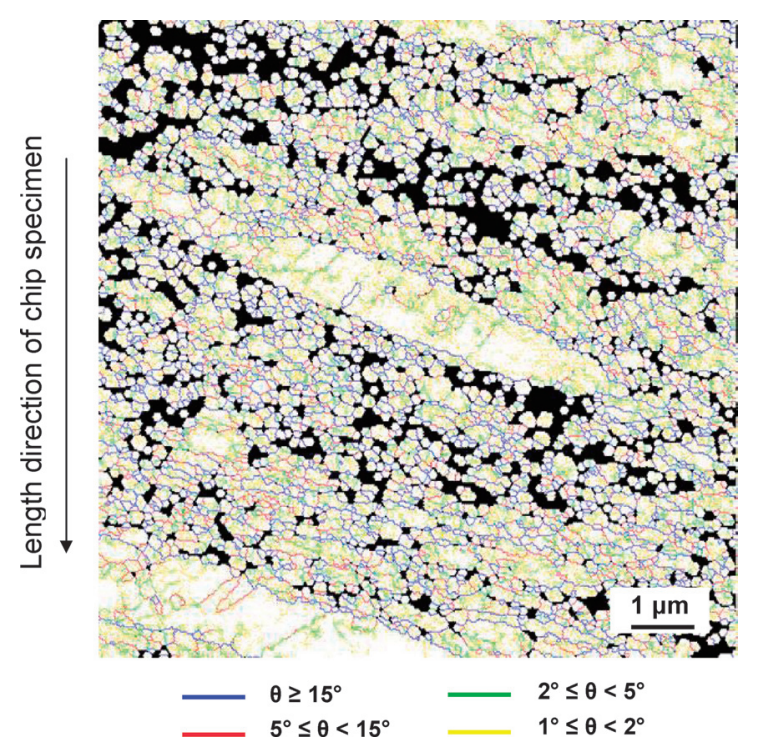

Fig. 8. Boundary misorientation map of the N5 chip specimen of the Inconel X-750 alloy. 

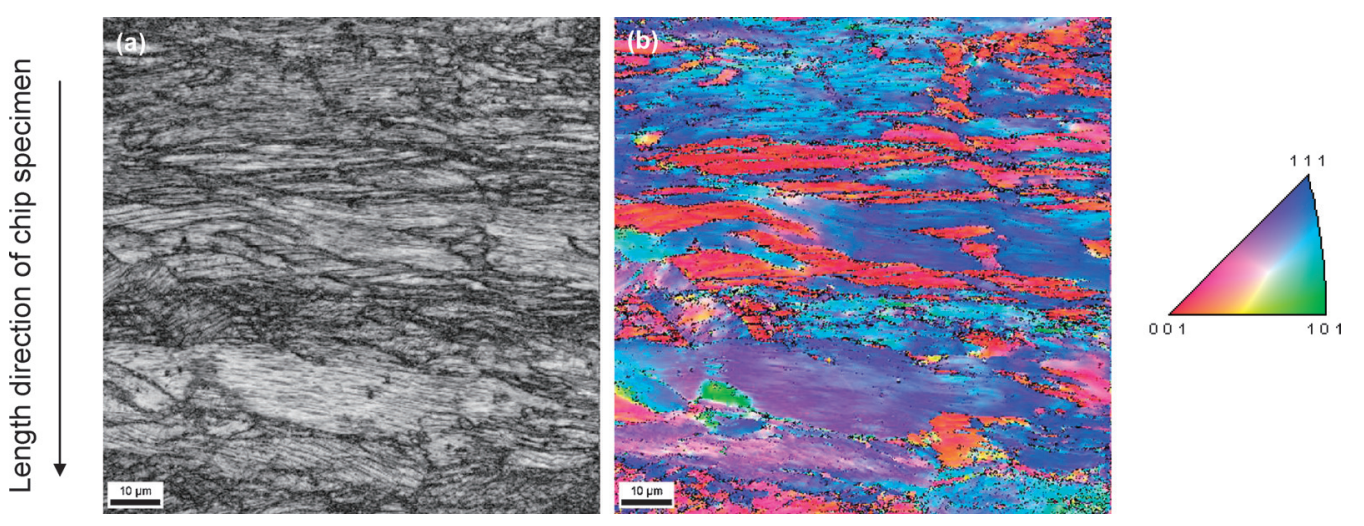

Fig. 9.

Image quality map and inverse pole figure map showing the crystal orientations parallel to $\mathrm{ND}$ of the chip specimen $(\gamma \approx 3)$ of the Inconel X-750 alloy annealed for $3.6 \mathrm{ks}$ at $973 \mathrm{~K}$.

(a) Image quality map, (b) inverse pole figure map (color coded map).

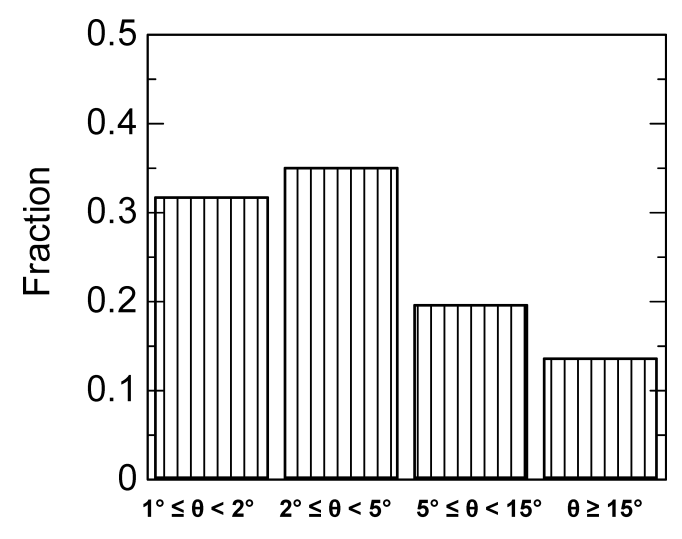

Misorientation $(\theta)$

Fig. 10. Fraction of grain boundaries with different misorientations $(\theta)$ in the chip specimen $(\gamma \approx 3)$ of the Inconel X-750 alloy annealed for $3.6 \mathrm{ks}$ at $973 \mathrm{~K}$.
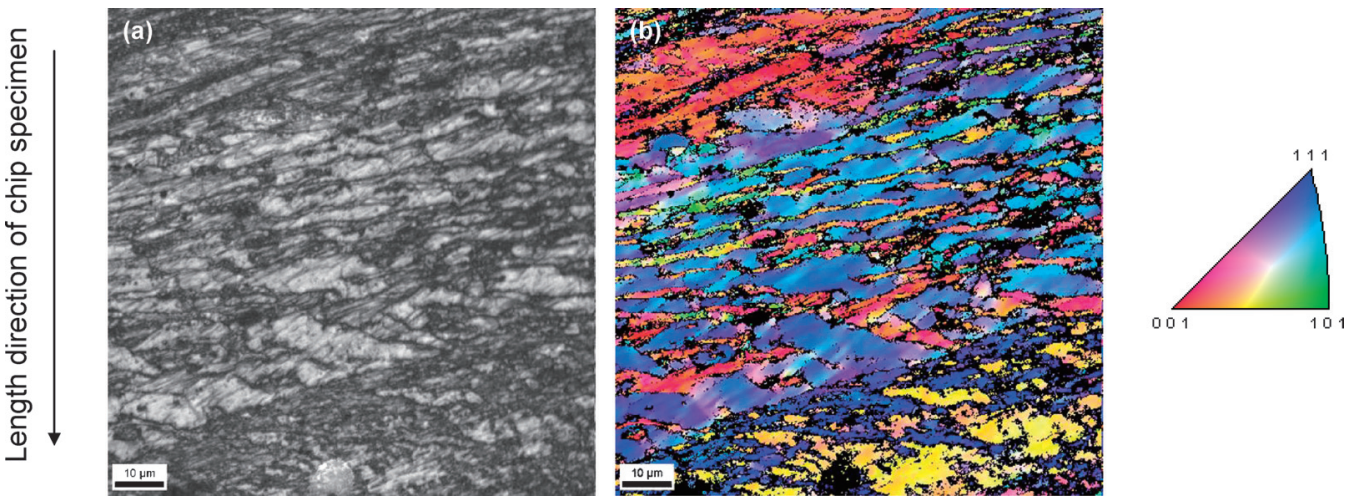

Fig. 11.

Image quality map and inverse pole figure map showing the crystal orientations parallel to ND of the A4 chip specimen $(\gamma \approx 4)$ of the 6061-T6 alloy.

(a) Image quality map, (b) inverse pole figure map (color coded map).
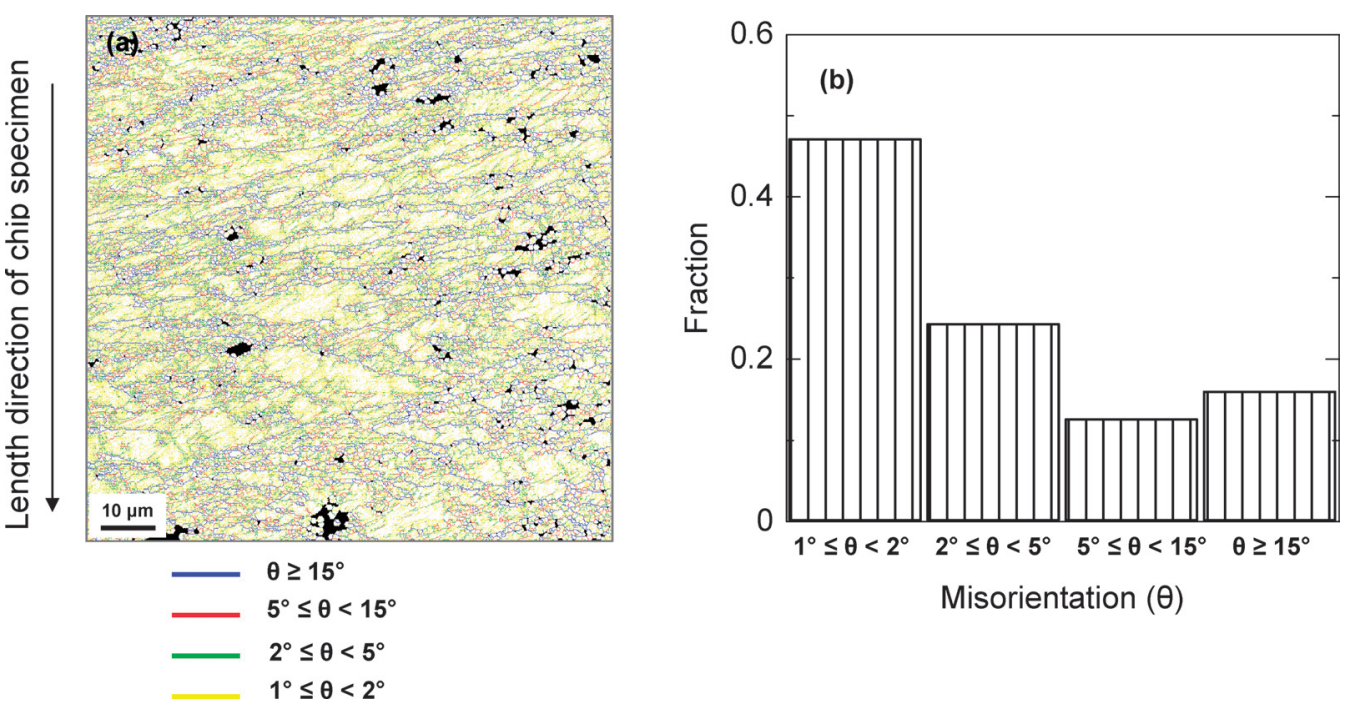

Fig. 12.

Boundary misorientation map and fraction of grain boundaries with different misorientations $(\theta)$ in the A4 chip specimen $(\gamma \approx 4)$ of the 6061-T6 alloy. (a) Boundary misorientation map, (b) fraction of grain boundaries with different misorientations $(\theta)$. 
in the Inconel X-750 alloy, many subgrains separated by small or medium angle grain boundaries $\left(\theta<15^{\circ}\right)$ are also visible within relatively large grains surrounded by large angle grain boundaries $\left(\theta \geq 15^{\circ}\right)$. According to the results of TEM observation of thin films at high magnifications, M. Ravi Shankar et al. ${ }^{18)}$ reported that the typical grain size was less than $100 \mathrm{~nm}$ in the chip specimens $(\gamma=3.2$ or 5.2) of the 6061-T6 alloy and that the average misorientation between grains was $\sim 20^{\circ}$ (large angle grain boundaries) in the chips of $\gamma=5.2$ and $\sim 10^{\circ}$ (medium angle grain boundaries) in the chips of $\gamma=3.2$. However, the fraction of large angle grain boundaries $\left(\theta \geq 15^{\circ}\right)$ is about $16 \%$, that of medium angle grain boundaries $\left(5^{\circ} \leq \theta<15^{\circ}\right)$ is about $13 \%$ and most of grain boundaries are small angle grain boundaries $\left(1^{\circ} \leq \theta<5^{\circ}\right)$ in A4 chip specimen $(\gamma \approx 4)$ (Fig. 12(b)). These results of the analysis are similar to those obtained on the chip specimens of the Inconel X-750 alloy described above. It seems to be necessary to examine a wide area by the FESEM/EBSP method in order to characterize quantitatively the microstructure of chip specimens.

The maximum shear strain $(\gamma)$ attained by machining was about 5 in the chip specimen of the Inconel X-750 alloy and about 4 in that of the 6061-T6 alloy in this study. The average misorientation between grains may depends on the shear strain of chip specimens and material properties. ${ }^{16-18)}$ Further study by the FESEM/EBSP method is needed for characterization of grain structures of chip specimens produced by machining in metals and alloys.

\subsection{Internal Friction and Hardness of Chip Specimens}

Figure 13 shows the relationship between internal friction $\left(Q^{-1}\right)$ and strain amplitude in the specimens of the Inconel X-750 alloy. In the N3 chip specimen $(\gamma \approx 3)$, internal friction increases from about 0.02 to 0.12 in the strain amplitude range from about $5 \times 10^{-6}$ to $9 \times 10^{-4}$, while the solution-treated specimen exhibits much smaller internal friction (from about 0.004 to 0.011 ) in the similar range of strain amplitude (from about $1.4 \times 10^{-5}$ to $2 \times 10^{-3}$ ). Internal friction of the N5 chip specimen $(\gamma \approx 5)$ increases from about 0.05 to 0.28 as the strain amplitude increases from about $4 \times 10^{-6}$ to $1.2 \times 10^{-3}$. These values of internal friction are larger than low carbon steels of high damping capacity, ${ }^{27)}$ ultra-fine grained $\mathrm{Al}^{9)}$ and $\mathrm{Ni},{ }^{10)}$ microcrystalline or nano-crystalline $\mathrm{Mg}^{28)}$ and $\mathrm{Mg}$ alloys, ${ }^{29)}$ and are comparable with metallic glasses. ${ }^{12,30)}$. Figure 14 shows the relationship between internal friction $\left(Q^{-1}\right)$ and strain amplitude in the specimens of the 6061-T6 alloy. Internal friction of the A4 chip specimen increases from about 0.020 to 0.065 as the strain amplitude increases from about $2 \times 10^{-6}$ to $7 \times 10^{-4}$, while that of the $\mathrm{A} 2$ specimen increases from about 0.012 to 0.047 with increasing strain amplitude from about $2 \times 10^{-6}$ to $1.2 \times 10^{-3}$. Internal friction of chip specimens is much larger than that of the as received specimens (about 0.0011 to 0.0069 in the strain amplitude range from about $3 \times 10^{-5}$ to $6 \times 10^{-3}$ ). In both alloys, internal friction of chip specimens tends to increase with increasing shear strain imposed by machining. The internal friction of chip specimens is larger in the Inconel X-750 alloy than in the 6061-T6 alloy. Thus, the effect of machining on the internal friction is different in materials.

As shown in Figs. 2 and 4, chip specimens contain many

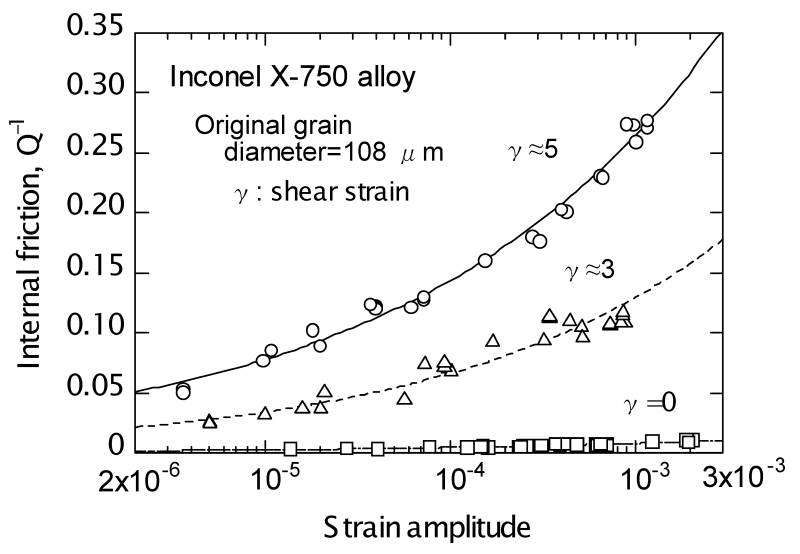

Fig. 13. Relationship between internal friction $\left(Q^{-1}\right)$ and strain amplitude in the specimens of the Inconel X-750 alloy.

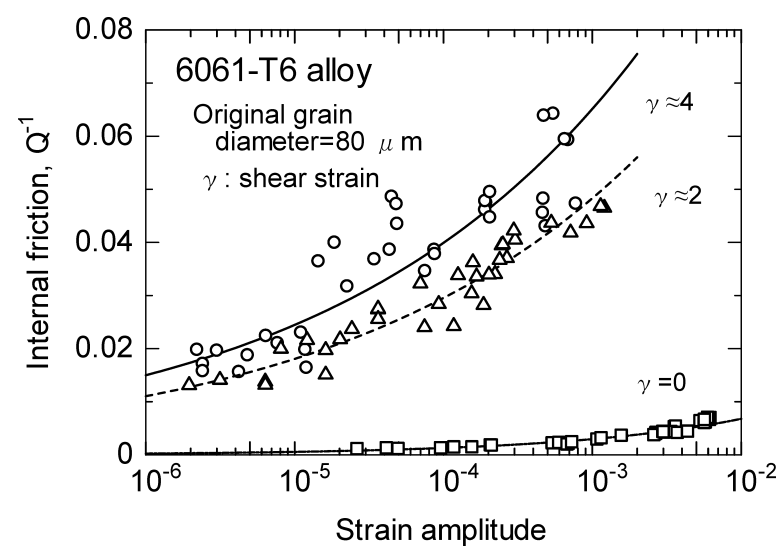

Fig. 14. Relationship between internal friction $\left(Q^{-1}\right)$ and strain amplitude in the specimens of the 6061-T6 alloy.

cracks lying transversely to the direction of chip formation (length direction) of specimens. It is known that internal friction of specimen is increased by initiation and growth of fatigue cracks during fatigue of stainless steels ${ }^{31)}$ and AZ91 magnesium alloy. ${ }^{32)}$ Göken and Riehemann ${ }^{32)}$ also reported that internal friction of fatigued specimens containing cracks increased with increasing strain amplitude for the measurement of internal friction in the magnesium alloy. These experimental results are similar to those obtained in this study. A large internal friction of chip specimen in this study is attributed principally to cracks formed by shear deformation during machining, although microstructures of chip specimens may affect the internal friction. Figure 15 shows the relationship between internal friction $\left(Q^{-1}\right)$ and strain amplitude in the chip specimen $(\gamma \approx 3)$ of the Inconel X-750 alloy annealed for $3.6 \mathrm{ks}$ at $973 \mathrm{~K}$. As will be described, the hardness of the chip specimen $(\gamma \approx 3)$ increased largely owing to fine precipitates of $\gamma^{\prime}$ phase or $\mathrm{M}_{23} \mathrm{C}_{6}$ carbide $^{24)}$ formed by the annealing. These fine precipitates may decrease internal friction of the chip specimen by pinning grain boundaries and dislocations. However, internal friction of the annealed chip specimens $(\gamma \approx 3)$ is almost the same as that of the chip specimen without annealing (Fig. 15). The experimental result may indicate that the origin of large internal friction in the chip specimens is not dislocation structures but cracks formed during machining. As listed in Table 2, cross section of chip specimen tends to decrease with increasing shear strain imposed in the ma- 


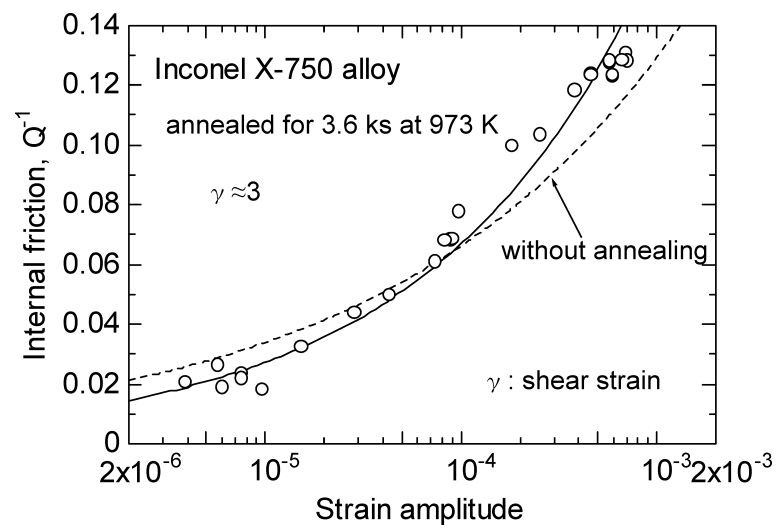

Fig. 15. Relationship between internal friction $\left(Q^{-1}\right)$ and strain amplitude in the chip specimen $(\gamma \approx 3)$ of the Inconel $X$ 750 alloy annealed for $3.6 \mathrm{ks}$ at $973 \mathrm{~K}$.

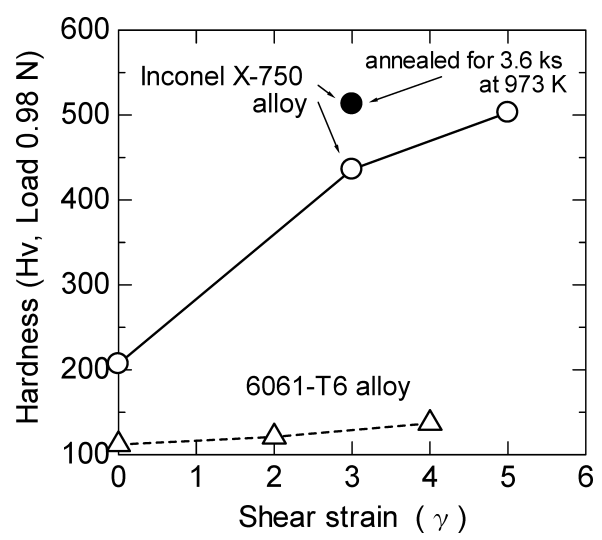

Fig. 16. Relationship between hardness and shear strain $(\gamma)$ of the specimens in the Inconel X-750 and 6061-T6 alloys.

chining. Effect of cracks on the internal fraction may become increasingly important as the cross section of chip specimen deceases. This may explain the experimental observation that internal friction of chip specimen increases with increasing shear strain. Further, initiation of cracks during machining may also depend on material properties.

Figure 16 shows the relationship between hardness and shear strain $(\gamma)$ of the specimens in the Inconel X-750 and 6061-T6 alloys. The hardness of the chip specimens is very high and increases from $436 \mathrm{Hv}$ to $503 \mathrm{Hv}$ in the Inconel X750 alloy as the shear strain increases from about 3 to 5 , although the hardness of solution-treated specimen is only $207 \mathrm{Hv}$. The hardness of the chip specimen $(\gamma \approx 3)$ increased further from $436 \mathrm{Hv}$ to $513 \mathrm{Hv}$ owing to fine precipitates of $\gamma^{\prime}$ phase or $\mathrm{M}_{23} \mathrm{C}_{6}$ carbide ${ }^{24)}$ formed by annealing for $3.6 \mathrm{ks}$ at $973 \mathrm{~K}$, although dislocation density is generally decreased by annihilation of dislocations during annealing. In contrast, the hardness of chip specimens was not so high compared with that of the as received specimen in the 6061-T6 alloy. The hardness is $121 \mathrm{Hv}$ for the A2 chip specimen $(\gamma \approx 2)$ and $137 \mathrm{Hv}$ for the A4 chip specimen $(\gamma \approx 4)$, whereas the hardness of the as received specimen is $112 \mathrm{Hv}$. The hardness value of A4 specimen $(\gamma \approx 4)$ is comparable with that of the chips of $\gamma=3.2(150 \pm 3 \mathrm{Hv})$ in the 6061-T6 alloy reported by M. R. Shankar et al. ${ }^{12)}$ Introduction of dislocations by machining may lead to an increase in the hardness of chip specimens, but the degree of hardness increase by machining depends on materials.
Chip specimens contain many cracks formed during machining, which are suppressed by other method such as ECAP, HPT and ARB. However, high hardness of chip specimens in the Inconel X-750 alloy indicates that each segment separated by cracks has high strength. Strong micro- or nano-crystalline particles can be produced from chips of some kinds of materials using a high energy ball mill. These particles will be potential strengthener for production of composite materials when incorporated in ductile metals or polymers.

\section{Conclusions}

Microstructures and properties were examined on the chip specimens of a given shear strain $(\gamma)$ that were produced by machining in the high-strength Ni-base Inconel X-750 alloy and 6061-T6 aluminum alloy. The misorientation between grains in chip specimens were examined by the electron backscatter diffraction pattern (EBSP) using a field emission scanning electron microscope (FESEM). The results obtained in this study are summarized as follows.

(1) Chip specimens involved many cracks lying transversely to the length direction (the direction of chip formation) formed during machining. Microstructures of the chip specimens were a deformation structure composed of elongated grains (a few $\mu \mathrm{m}$ to about $100 \mu \mathrm{m}$ in length and about $1 \mu \mathrm{m}$ to a few tens $\mu \mathrm{m}$ in width for the chip specimen of $\gamma \approx 3$ of the Inconel X-750 alloy, and a few $\mu \mathrm{m}$ to a few tens $\mu \mathrm{m}$ in length and about $1 \mu \mathrm{m}$ to $10 \mu \mathrm{m}$ in width for the chip specimen of $\gamma \approx 4$ of the 6061-T6 alloy). In the case where detailed analysis by FESEM/EBSP was possible, the fraction of large angle grain boundaries with misorientation $(\theta)$ equal to or larger than $15^{\circ}\left(\theta \geq 15^{\circ}\right)$ and that of medium angle grain boundaries $\left(5^{\circ} \leq \theta<15^{\circ}\right)$ was relatively small, and most of grain boundaries were small angle grain boundaries $\left(1^{\circ} \leq \theta<5^{\circ}\right)$ in the chip specimens of both alloys. Many subgrains separated by small or medium angle grain boundaries $\left(\theta<15^{\circ}\right)$ were also visible within large grains surrounded by large angle grain boundaries $\left(\theta \geq 15^{\circ}\right)$. The fractions of large $\left(\theta \geq 15^{\circ}\right)$ and medium $\left(5^{\circ} \leq \theta<15^{\circ}\right)$ angle grain boundaries increased a little by annealing for $3.6 \mathrm{ks}$ at $973 \mathrm{~K}$ in the chip specimen of $\gamma \approx 3$, although most of grain boundaries were small angle grain boundaries $\left(1^{\circ} \leq \theta<5^{\circ}\right)$.

(2) The internal friction of the chip specimens was much larger than that of the solution-treated or as received specimens in the Inconel X-750 and 6061-T6 alloys. For example, in the Inconel X-750 alloy, the internal friction of the chip specimen of shear strain $\gamma \approx 5$ increased from about 0.05 to 0.28 with increasing strain amplitude from about $4 \times 10^{-6}$ to $1.2 \times 10^{-3}$, while internal friction of the solution-treated specimen changed from about 0.004 to 0.011 in the strain amplitude range from about $1.4 \times 10^{-5}$ to $2 \times$ $10^{-3}$. Similar results were obtained on the chip specimens of the 6061-T6 alloy, although the values of internal friction in the chip specimens of the 6061-T6 alloy were smaller than in those of the Inconel X-750 alloy. Such a large value of internal friction in chip specimens was attributed principally to many cracks formed by shear deformation during machining.

(3) The hardness of the chip specimens was very high 
in the Inconel X-750 compared with the as solution-treated specimen. For example, the hardness was $436 \mathrm{Hv}$ in the specimen of $\gamma \approx 3$ and $503 \mathrm{Hv}$ in the one of $\gamma \approx 5$ in the Inconel X-750 alloy, while the hardness of the as solutiontreated specimen was only $207 \mathrm{Hv}$. The hardness of the specimen of $\gamma \approx 3$ increased to $513 \mathrm{Hv}$ by annealing for $3.6 \mathrm{ks}$ at $973 \mathrm{~K}$. In contrast, the hardness of chip specimens was not so high compared with that of the as received specimen in the 6061-T6 alloy. The degree of hardness increase by machining depended on materials.

\section{REFERENCES}

1) V. M. Segal, V. I. Reznikov, A. E. Drobyshevskiy and V. I. Kopylov: Russ. Metall. (Metally), 1 (1981), 99.

2) Z. Horita, M. Furukawa, T. G. Langdon and M. Nemoto: Materia Jpn., 37 (1998), 767.

3) M. Kamachi, M. Furukawa, Z. Horita and T. G. Langdon: Mater. Sci. Eng. A, A347 (2003), 223.

4) R. Z. Valiev, Y. Estrin, Z. Horita, T. G. Langdon, M. J. Zehetbauer and Y. T. Zhu: JOM (J. Met.), 58 (2006), No. 4, 33.

5) Y. Saito, H. Utsunomiya, N. Tsuji and T. Sakai: Acta Mater, 47 (1999), 579

6) B. Q. Han, E. J. Lavernia and F. A. Mohamed: Metall. Mater. Trans. A, 35A (2004), 1343.

7) H. W. Höppel and R. Z. Valiev: Z. Metallkd., 93 (2002), 641.

8) R. R. Mulyukov, N. A. Akhmadeev, R. Z. Valiev and S. B. Mikhailov: Mater. Sci. Eng. A, A171 (1993), 143.

9) Y. Koizumi, M. Ueyama, N. Tsuji, Y. Minamino and K. Ota: J. Alloy Comp., 355 (2003), 47.

10) Y. Koizumi, M. Ueyama, N. Tsuji, Y. Minamino and K. Ota: J. Jpn. Inst. Met., 69 (2005), 997.

11) K. Sugimoto: Tetsu-to-Hagané, 60 (1974), 2203.

12) H. Mizubayashi, Y. Ishikawa and H. Tanimoto: Mater. Sci. Eng. A, A370 (2004), 546.

13) Y. Huang and T. G. Langdon: J. Mater. Sci., 37 (2002), 4993.
14) A. Vinogradov, S. Hashimoto, V. Patlan and K. Kitagawa: Mater. Sci. Eng. A, A319-321 (2001), 862.

15) E. Ma: JOM (J. Met.), 58 (2006), No. 4, 49.

16) T. L. Brown, S. Swaminathan, S. Chandrasekar, W. Dale Compton, A. H. King and K. P. Trumble: J. Mater. Res., 17 (2002), 2484.

17) S. Swaminathan, M. R. Shankar, S. Lee, J. Hwang, A. H. King, R. F. Kezar, B. C. Rao, T. L. Brown, S. Chandrasekar, W. Dale Compton and K. P. Trumble: Mater. Sci. Eng. A, A410-411 (2005), 358.

18) M. Ravi Shankar, S. Chandrasekar, W. Dale Compton and A. H. King: Mater. Sci. Eng. A, A410-411 (2005), 364.

19) B. L. Adams, S. I. Wright and K. Kunze: Metall. Trans. A, 24A (1993), 819.

20) D. J. Prior, A. P. Boyle, F. Brenker, M. C. Cheadle, A. Day, G. Lopez, L. Peruzzo, G. J. Potts, S. Reddy, R. Spiess, N. E. Timms, P. Trimby, J. Wheeler and L. Zetterström: Am. Mineral., 84 (1999), 1741.

21) T. Baumeister (ed.): Marks' Standard Handbook for Mechanical Engineers, 7th ed., McGraw-Hill, Kogakusha, Tokyo, (1967), 13.

22) N. Takenaka: Mechanical Technology (2), revised edition, Corona, Tokyo, (2001), 8.

23) R. De Batist: Internal Friction of Structural Defects in Crystalline Solids, North-Holland American Elsevier, New York, (1972), 347, 419.

24) S. Watanabe: J. Jpn. Soc. Heat Treat., 31 (1991), 248.

25) H. Ni and A. T. Alpas: Mater. Sci. Eng. A, A361 (2003), 338.

26) C. T. Sims and W. C. Hagel: The Superalloys, John Wiley \& Sons, New York, (1972), 52.

27) M. Takahashi, A. Okamoto and H. Iida: Trans. Iron Steel Inst. Jpn., 20 (1980), 279.

28) Z. Trojanová, P. Lukáč, H. Ferkel and W. Riehemann: Mater. Sci. Eng. $A, \mathbf{A 3 7 0}$ (2004), 154.

29) V. N. Chuvil'deev, T. G. Nieh, M. Yu. Gryaznov, A. N. Sysoev and V. I. Kopylov: Scr. Mater, 50 (2004), 861.

30) Y. Hiki, T. Yagi, T. Aida and S. Takeuchi: Mater. Sci. Eng. A, A370 (2004), 302.

31) H. Fujita, M. Tanaka and O. Kamiya: Tetsu-to-Hagané, 67 (1981), 1815.

32) J. Göken and W. Riehemann: Mater. Sci. Eng. A, A370 (2004), 417. 